\title{
Research Paper \\ Relationship Between Neuropsychological and Physical Environmental Perception in Patients With Dementia and Alzheimer Disease
}

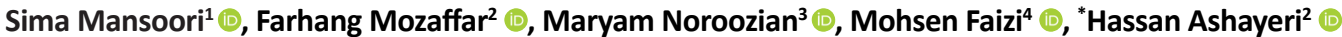

1. PhD. in Architecture, Department of Architecture, School of Architecture and Environmental Design, Iran University of Science and Technology, Tehran, Iran.

2. Associate professor in Architecture, Department of Architecture, School of Architecture and Environmental Design, Iran University of Science and Technology, Tehran, Iran

3. Neurology Division, Department of Psychiatry Tehran University of Medical Sciences, Tehran, Iran.

4. Professor, Architecture, Department of Architecture, School of Architecture and Environmental Design, Iran University of Science and Technology, Tehran, Iran.

5. Professor, Neuropsychology and psychiatry, Rehabilitation of Iran University of Medical science, Tehran, Iran.

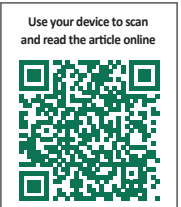

Citation Mansoori S, Mozaffar F, Noroozian M, Faizi M, Ashayeri H. [Relationship Between Neuropsychological and Physical Environmental Perception in Patients With Dementia and Alzheimer Disease (Persian)]. Iranian Journal of Psychiatry and Clinical Psychology. 2019; 24(4):426-443. http://dx.doi.org/10.32598/ijpcp.24.4.426

http://dx.doi.org/10.32598/ijpcp.24.4.426

Received: 27 Feb 2018

Accepted: 22 Jul 2018

Available Online: 01 Jan 2019

Keywords:

Perception, Physical environment, Dementia, Alzheimer disease, Neuropsychology

\section{A B S T R A C T}

Objectives The current study examines the features of the physical environment affecting environmental perception and tries to explain paradigm of architecture based on the neuropsychological features. This paper investigates the relationship between the two aspects of the physical and neuropsychological environment in the patients with dementia Alzheimer's type in order to meet the needs of the mental processes (sensation, perception, memory, and the organization of targeted behavior and adaptation to the environment for executive functions).

Methods This study was conducted by inductive content analysis method based on 9 semi-structured interviews and inductive content analysis from related literature.

Results Environmental perception was found as category based on two inductive content analyses. Moreover, 28 concepts of first level concepts (coding) and three concepts of "indifference to the elements in the physical environment", "willingness to attend familiar places" and "stressors in unfamiliar places" as subcategories were obtained from semi-structured interviews.

Conclusion Explaining the features of the physical environment combined with neuropsychological environment can play a significant role in creating the habitat, care and treatment environment of these patients. Therefore, the sensory cue of the environment is effective in reducing behavioral disorders. Also, the perception of a more powerful environment means a greater interaction between these two dimensions of the environment.

\section{Extended Abstract}

\section{Introduction}

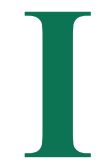

ncreased longevity and aging are among the most significant risk factors of catching Alzheimer Disease (AD). Recent studies have investigated the effects of physical and neuropsychological factors on the environmental perception of with the patients with AD. However, data are scarce on the associations between the main features of the physical environment and brain activities of Ad patients [1].

\section{Method}

This was a descriptive analytical study, which was performed using inductive content analysis. The final

* Corresponding Author:

Hassan Ashayeri, PhD.

Address: Department of Psychiatry, School of Medicine, Tehran University of Medical Sciences, Tehran, Iran.

Tel: +98 (21) 2228052

E-mail: ashayerih.neuroscientist@yahoo.com 
outcomes were the result of the interpretation and combination of semi-structured interviews with the people with dementia $\mathrm{AD}$ type and their caregivers and review of the literature in this regard. In addition, the similarities and differences between the qualitative physical features of the environment were assessed from the viewpoint of the patients with $\mathrm{AD}$, their caregivers, and based on the literature review. The study data were collected through semi-structured interviews (15-30 minutes) using Mild Cognitive Impairment (MCI) and Functional Assessment Staging (FAST 4,5). In total, 9 participants were enrolled in the study, including four patients with $\mathrm{AD}$ and five caregivers. Furthermore, the required data were extracted from the current literature. The interviews were entitled Participants with 'AD (PAD)' and 'Caregiver Participants (CGP)'.

\section{Results}

The main theme emerging from the interviews was the perceptions of patients with AD in a Yadman Clinic in Tehran, Iran. The results of the content analysis of the interviews revealed the main perceptions of the patients with AD (Table 1). In addition, the inductive content analysis of the literature indicate that environmental perception has the most significant effect on the sense of place in these patients. Various aspects of the memory (e.g. spatial and topographic memory) were also observed to be effective in this regard (Table 2).

\section{Discussion}

The conceptual memory of the patient interferes with the memory of the spatial elements (place, light, and sound) [16]. Therefore, the fading of memories is accompanied by the loss of the sense of space and spatial awareness. With this background in mind, the patient may feel stressed in unfamiliar places due to the loss of the spatial memory. This finding is consistent with the contents of the live interviews in the present study.

The Parahippocampal Cortex (PHC) is activated more significantly while encountering new perspectives as compared to repetitive scenes and complex environments. According to the experiments, PHC shows a stronger response to spaces and places compared to the other visual stimulants [19]. According to Ziesel, spatial features play a key role in developing the spatial memories in patients with AD [20]. Furthermore, Lee and Wilson do not consider the spatial memory to be created by separate places, but rather, they believe that it is formed by the connections between a set of places [21]. Therefore, the contents obtained from the analysis of the interviews with the patients and their caregivers in the present study indicated the concept of "willingness to attend familiar places" and "creating proper spatial cognition in these environments" based on the identified features in the analysis.

The spatial memory of familiar places could remarkably improve the spatial memory in the elderly individuals [23], which is one of the similarities between the content analyses in the current research. According to Bongs, the sense of place is influenced by the physical space and spatial activities, which form the perceptions of individuals toward the environment [22]. This finding denotes another similarity between the results of the content analyses in our study. Therefore, prominent stimulants in the environment lead to the sustainability and stability of a strong sense of space in the hippocampus. Moreover, these stimulants develop specific memory pathways through the prominent spatial features. Likewise, the findings of Stenberg and Wilson demonstrated an association between the spatial memory and sense of space, so that the memories formed in the space influence the sense of place in individuals [18].

Environmental perceptions are involved in interpreting the perceptual-spatial data, laying the ground for the realization and organization of purposeful adaptive behaviors. Other benefits of such approaches include the reduction of stress and anxiety and overcoming memory deficiencies. Therefore, it could be concluded that stronger environmental perception is synonymous with the more frequent interactions of various aspects of physical environments and neuropsychological components. Recognition of the qualitative dimensions of the physical environment is essential to further influence neurological and brain functions, which in turn increase excitation and maintain attention. Enriching of the components of qualitative environmental perception of patients with AD with the help of the lived memory could play a pivotal role in their daily performance.

\section{Ethical Considerations}

\section{Compliance with ethical guidelines}

All ethical principles were considered in this article. The participants were informed about the purpose of the research and its implementation stages; They were also assured about the confidentiality of their information; Moreover, They were allowed to leave the study whenever they wish, and if desired, the results of the research would be available to them. 
Table 1. Content analysis of the interview from patients with $\mathrm{AD}$ and caregivers

\begin{tabular}{llc}
\hline Meaning Unit & First Level of Concept Coding & $\begin{array}{c}\text { Second Level of } \\
\text { Concept }\end{array}$ \\
(Subcategory) & $\begin{array}{c}\text { Third Level of } \\
\text { Concept } \\
\text { (Category) }\end{array}$ \\
\hline
\end{tabular}

How do you feel in the park?

P1AD: I feel nothing. Sometimes I sit, walk, and then return

home. I do not feel any different in the two places.

Where do you feel at peace?

CGP3: I do not feel any difference in any place.

In which areas of the home do they feel better?

CGP2: It makes no difference. There was a garden that used to

amuse them, but they show no interest in that now and are

mostly indifferent.

So, what do you think the domestic environment means to

them?

P3AD: They are just indifferent.

The other day, I actually slipped on the stairs and fell down when

I was outside because I am not focused; my head is in the clouds.

Besides loneliness, what else do you feel at home?

P4AD: Nothing, I do not feel anything at home.

What if the physical environment changes? Would that make any difference?

Not really.

Where else do you go except for your home?

Nowhere; Months pass by and I do not go anywhere.

Are there any places you have been to and liked?

No.

Have you ever been to any places where you were disturbed and would never like to go back?

Not at all. There are so many of them that I hardly think about it.

Indifference to the place of residence

Indifference to the elements in the environment

Lack of attention to the elements in the environment

Inadequate sensitivity to the physi-

cal environment of the residence

Lack of attention to the physical environment

Lack of attention to the environment

Lack of motivation toward the elements in the environment

You mean you do not think about the environment? What attracts your attention in different places?

Nothing.

Is there anything in this room now that interests you? Or you dislike?

No.

How do you feel about this place? GCP5: Nothing special.

Are there any particular places at home that you like?

No.

How does he/she feel at home?

$\mathrm{He} / \mathrm{she}$ has been really unmotivated lately and sleeping most of the time. 


\section{Meaning Unit}

In what places do you think your father would feel most comfortable?

CGP3: The most peaceful places are home, the neighborhood, and a park nearby. No matter where else we take him, even the best places, he never feels comfortable and wants to come back home immediately; he just wants to be at home. Home is his safe haven.

Does he go to the park by himself?

Yes. He never gets lost except in the places he is not familiar with. He knows where the park is. If he does not know a place, he never stays, not even for a single minute. He has the park on his mind; when we had gone to Mashhad, he was lost once and looked for the park.

Where else does his mind work properly?

P2AD: He also likes the mosque and the high school where he was a teacher.

Is his thinking disturbed while shopping?

CGP1: No, because we do our shopping in particular places that he is familiar with.

CGP2: He has no interest in television, and we only read the religious books that he used to read to him, and he listens.

Has he ever been disturbed in any environments?

CGP4: He is normally disturbed in crowded places and when people try to talk to him all the time. For examples, he becomes stressed in unfamiliar wedding parties.

Apart from crowded places, are there any other factors that might disrupt his perception?

No, only crowded places and stress. Sometimes we suggest taking him to the park, but he disagrees, or we go to my daughter's place, but he wants to leave immediately.

PAD3: Noise and crowds really get on my nerves. Although I love my grandchildren and children, I am no longer in the mood when they come to visit. But I still love children.

What kinds of environments stress him out? CGP1: New and unfamiliar places, official places, I dare say the things that may or may not be done.

In which environments do you think he is most likely to be disturbed?

CGP2: Where his colleagues might be or family gatherings where everyone talks, and also in crowds.

Are there any environments that particularly disrupt his perception?

CGP3: Not really, but if he gets out of the safe atmosphere of the house or neighborhood, he will feel restless.

CGP5: Where it is less crowded, I try to take him out anyways, but there is always noise, music, and children in public places, and he gets impatient.
Home as a peaceful shelter Feeling secure at Home Willingness to attend familiar places

Proper spatial cognition in familiar environments (pre-experienced) (proper consciousness in religious places [mosques], cognitive ability [attention] in familiar [religious] subjects, lack of attention to new subjects, good memories of former workplaces, feeling of success in former workplaces, willingness)
Stressors in unfamiliar places Stressors in new professional environments,

Unfamiliarity with the environment due to loneliness

(Discomfort and restlessness in unfamiliar places, unwillingness to attend crowded places: impatience and discomfort in crowded places,

stress and anxiety in crowded places, loss of concentration in new environments, unwillingness to attend unfamiliar places, intolerance of unfamiliar places [willingness to return to familiar places]) 
Table 2. Content analysis of literature: Relationship between Physical and Neuropsychological Environment Components

\begin{tabular}{|c|c|c|c|}
\hline Category & Subcategory & $\begin{array}{l}\text { Influenced Components of the Neuro- } \\
\text { logical Environment }\end{array}$ & $\begin{array}{c}\text { Influential Components of the Physical } \\
\text { Environment }\end{array}$ \\
\hline \multirow{2}{*}{$\begin{array}{l}\text { Environmental } \\
\text { perception }\end{array}$} & Sense of place & Memorable effective spatial qualities & $\begin{array}{c}\text { New scenes, detailed space, new envi- } \\
\text { ronmental elements, memorable places, } \\
\text { a series of related places }\end{array}$ \\
\hline & Deep sense & $\begin{array}{c}\text { Cognitive map, Topographic memory, } \\
\text { Creating effective spatial qualities in visu- } \\
\text { al-spatial memory }\end{array}$ & $\begin{array}{l}\text { Familiar and friendly environments, easy } \\
\text { way finding, sensory stimuli in the envi- } \\
\text { ronment, multisensory environments }\end{array}$ \\
\hline
\end{tabular}

PSYCHIATRYAND CUNICAL PSYCHOLOGY

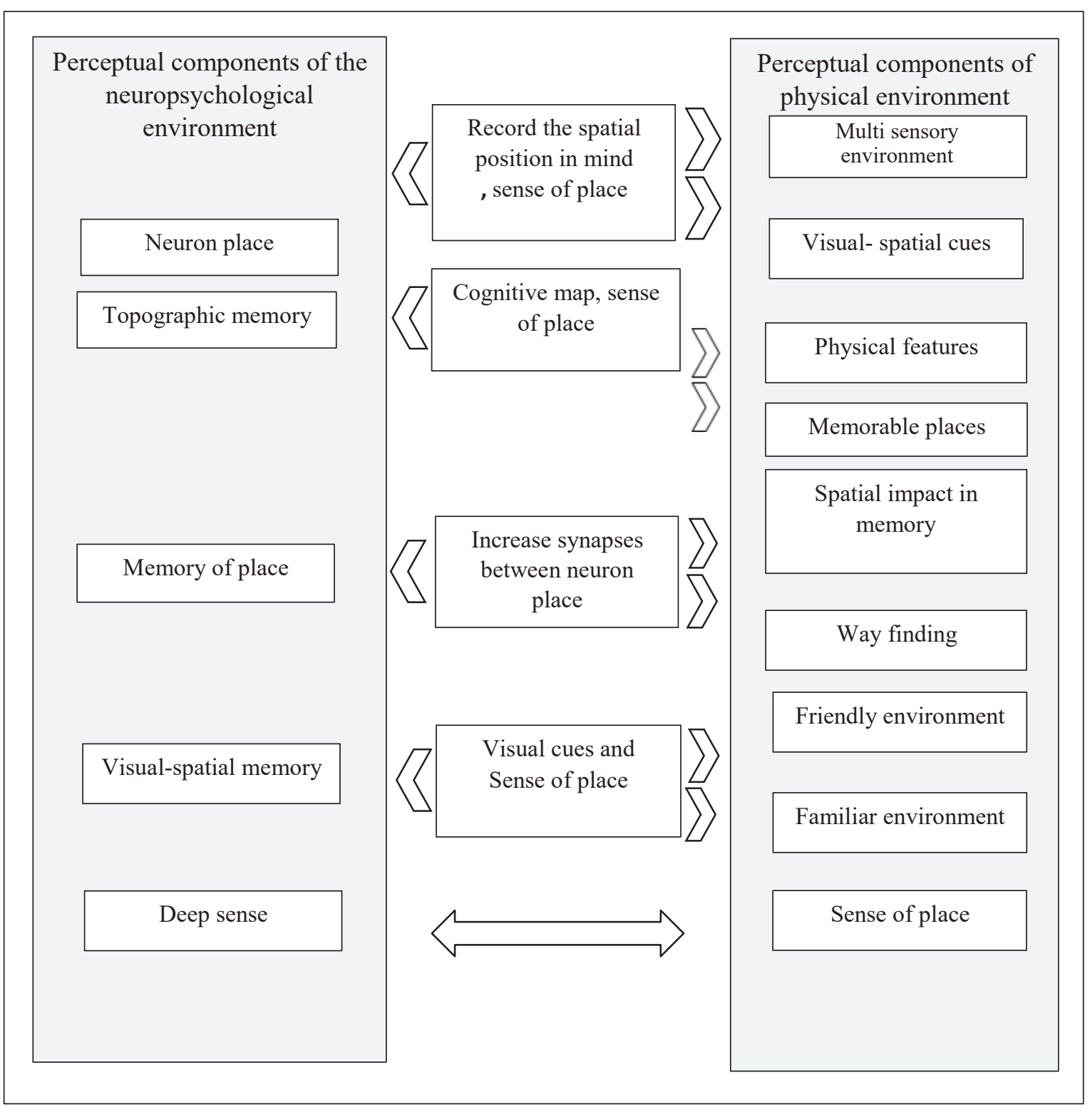

Iranian Journal of

Figure 1. The relationship between physical and neuropsychology in environmental perception for patient with $\mathrm{AD}$ 
Funding

This research was extracted from the $\mathrm{PhD}$. thesis of the first author in the School of Architecture and Environmental Design, Iran University of Science and Technology.

\section{Authors contributions}

The authors contributions is as follows: Conceptualization: Sima Mansoori, Farhang Mozaffar, and Mohsen Faizi; Investigation and analysis: Maryam Noroozian and Hassan Ashayeri; and editing and drafting: Sima Mansoori and Maryam Noroozian.

\section{Conflict of interest}

The authors declare no conflict of interest.

\section{Acknowledgements}

We appreciate staff and participants of Yadman Clinic of Neurology for dementia AD type who helped us with this research. 
This Page Intentionally Left Blank 


\title{
تبيين ويزُكَىهاى محيط كالبدى و عصبروانشناختى در ادراك محيطى مبتلايان به دمانس از نوع آلزايمر تبين
}

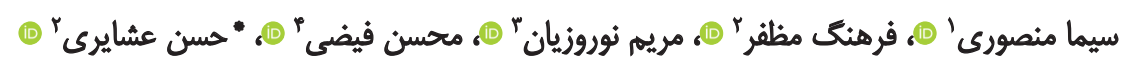

ا- د دكتراى معمارى، كروه معمارى، دائشكده معمارى و شهرسازى، دائشكاه علم و صنعت ايران، تهران، ايران.

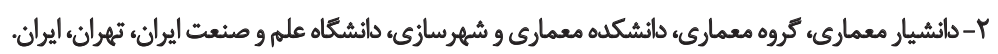

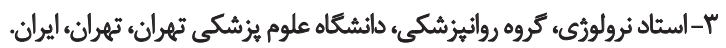

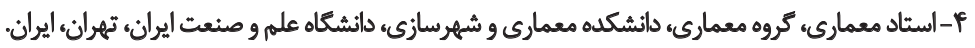

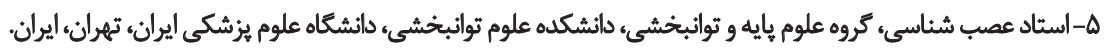

\begin{abstract}
حكيد

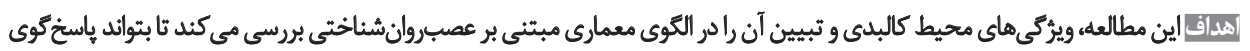

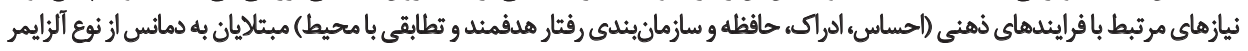

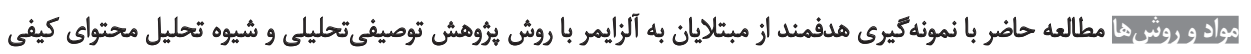

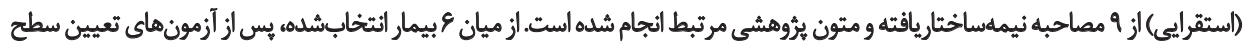

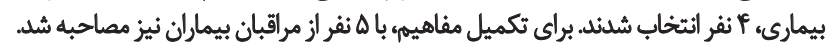

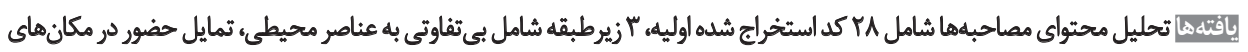

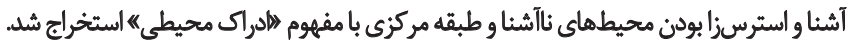

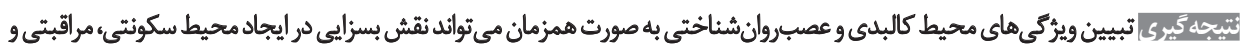

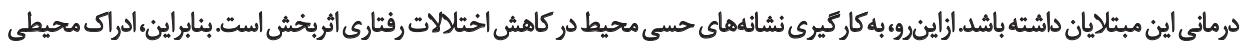

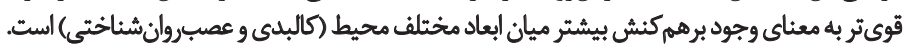

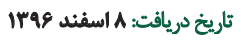

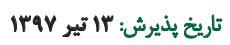

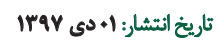

كليدواثهها: ادراك، محيط كالبدى، البيد، دمانس، آلزايمر، عصبروانشُناختئي

از زنوع آلزايمر، انجام شده است. بهطور خاص، اطلاعات اندكى در رابطه مقلمه

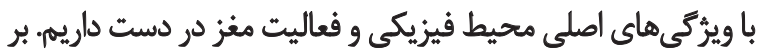

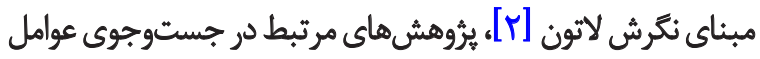

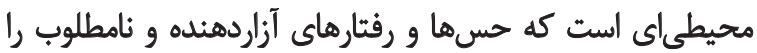

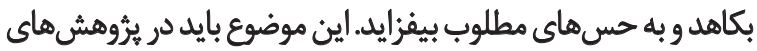

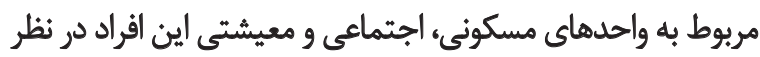
كرفته شود. اين مسئله تاكنون بررسى نشانده است

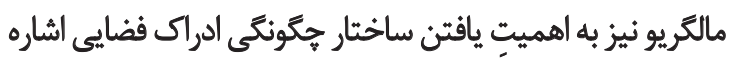

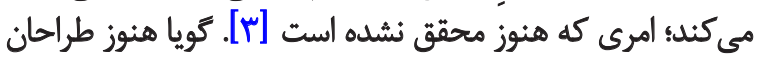

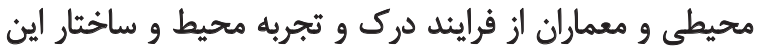

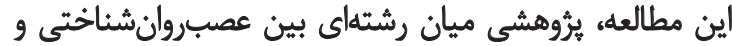

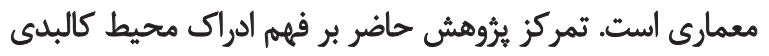

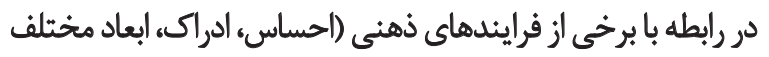

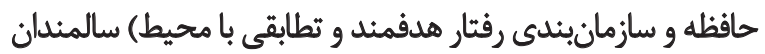

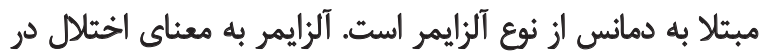

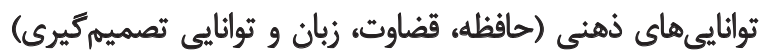

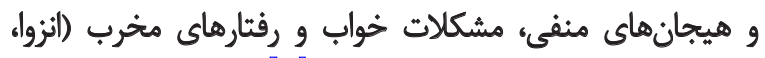

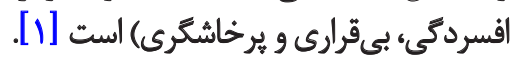

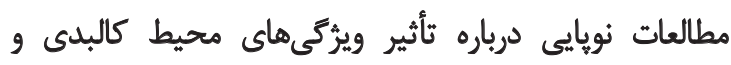

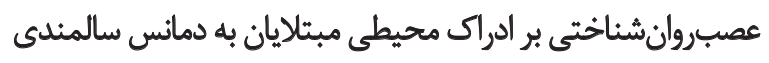
بshayerih.neuroscientist@yahoo.com بلفت الكترونيكي: 
محر مائه بودن مصاحبه ها به هر يك از مشاركت كنيندكان توضيح داده

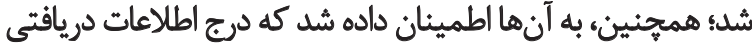

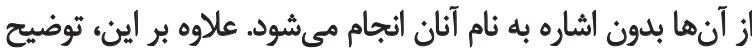

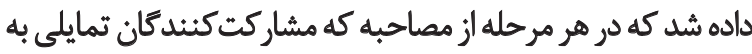

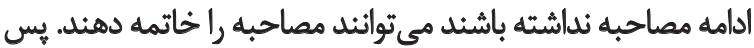

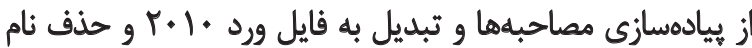

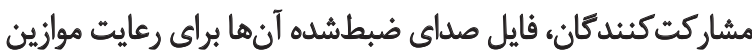

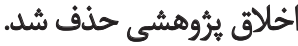

در اين يروهش از روش توصيفىتحليلى با شيوه تحليل محتواى

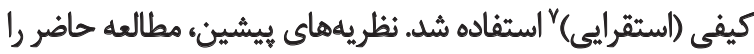

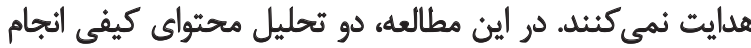

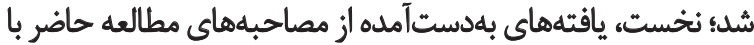

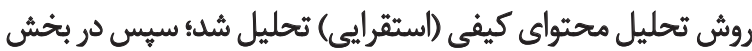

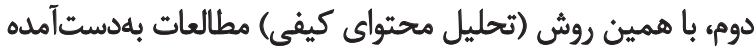

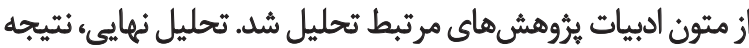

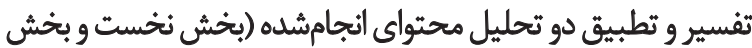

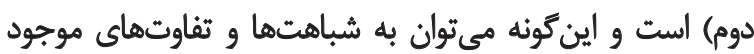

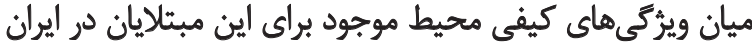

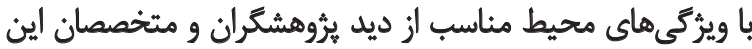

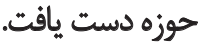

تحليل محتواى كيفى، روشى مؤثر در توصيف و طبقهبندي

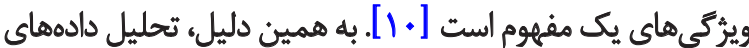

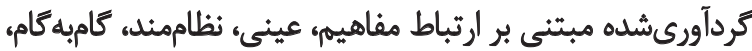

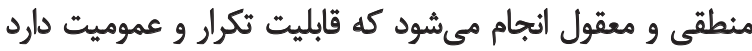

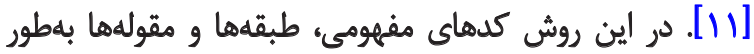

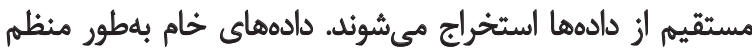

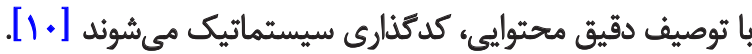

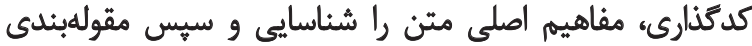

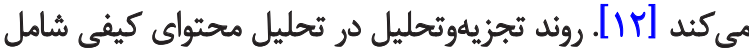

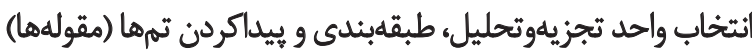

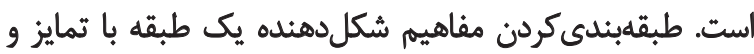

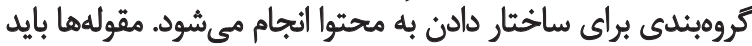

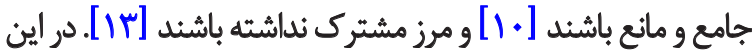

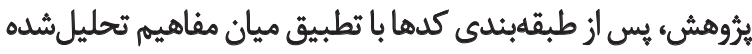
در زيرطبقات، مفهوم اصلى به دست ميندآيد

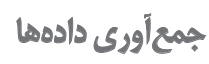

دراين يروهش، ابتداباروش مصاحبه نيمهاخسارياريافتهاز مبتلايانى

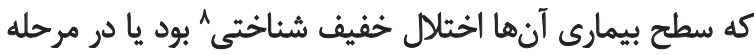

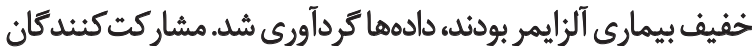

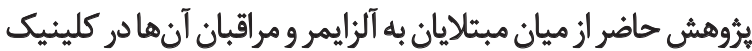

7. Inductive content analysis

8. Mild Cognitive Impairment (MCI)
تجربه توسط مبئلايان به دمانس از نوع آلزايمر' بيىاطلاع هستندي

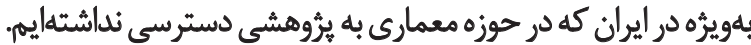

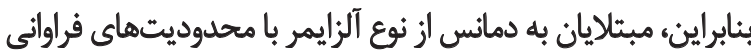

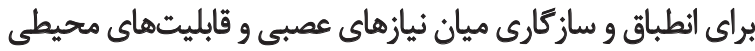

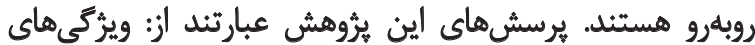

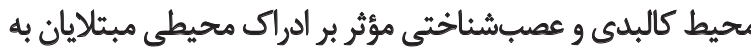

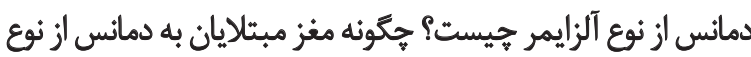

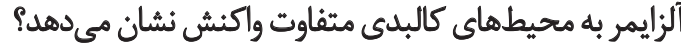
يكى از مشكلات اين مبتلايان حواسيرتى است. بروكى و بوهلين"،

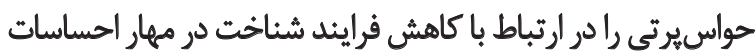

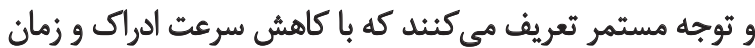

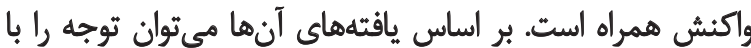

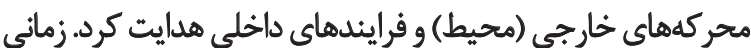

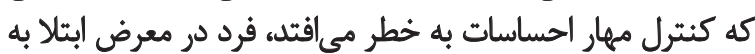
تداخل حسى و حواسيرتى قرار مي كيرد [F] كايلان و برمن "در نظريه حفظ توجه با بمك فرايندهاى تسهيلى

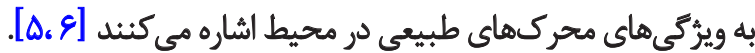

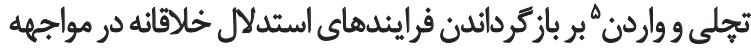

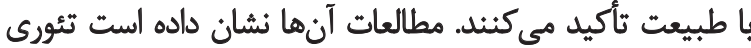

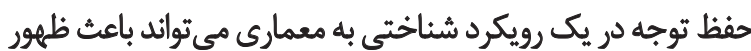

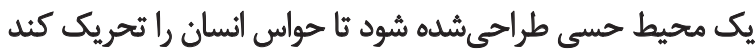

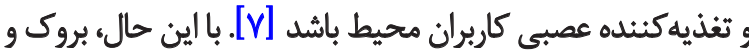

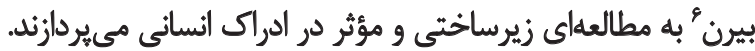

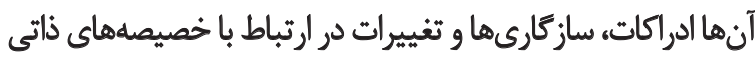

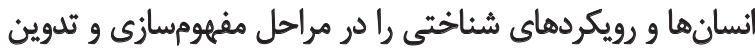

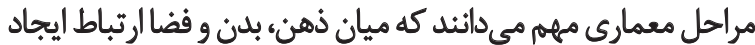

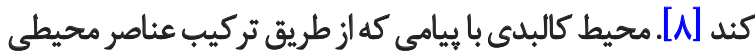

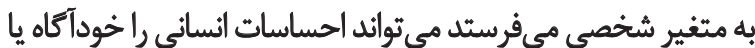

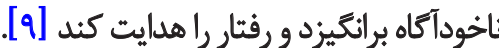

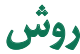
در اين يرؤش براى رعايت موازين اخلاقى يرؤهش (محرمانه

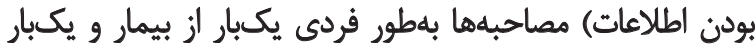

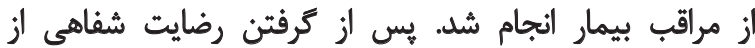

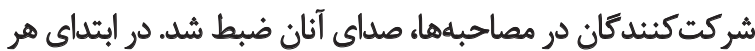

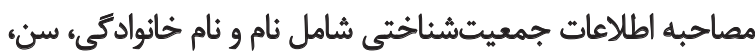

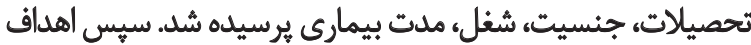

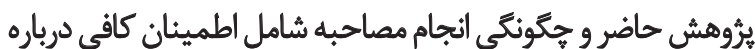

1. Dementia Alzheimer type

2. Brocki \& Bohlin

3. Kaplan \& Berman

4. Attention Restoration Theory (ART)

5. Atchley \& Warden

6. Brooke \& Biren 


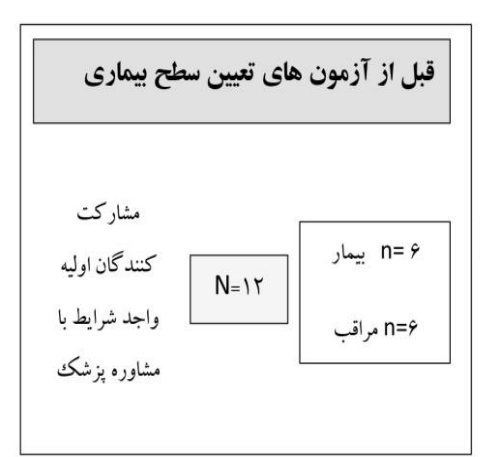

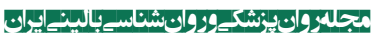

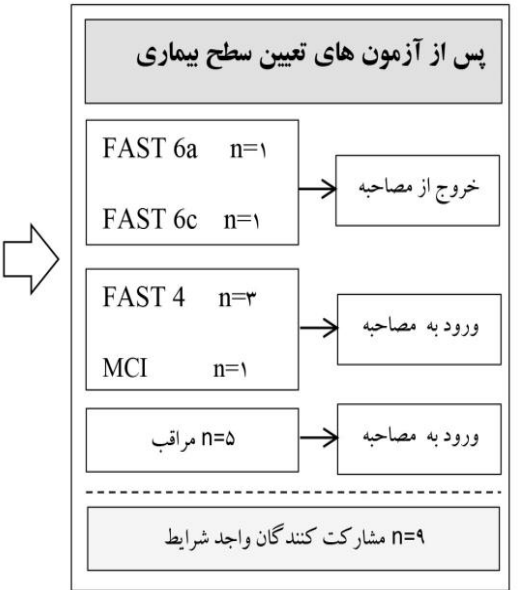

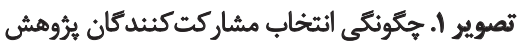
FAST: Functional Assesment Staging Test; MCl: Mild Cognitive Impairment; n: تعداد مشاركت كثئدكًان در هر مرحله؛ N: تعداد كل مشاركت كنئدكان اوليه

هصاحبه با سؤالي بازٔ وكلي درباره احساس هشاركت كنئله نسبت

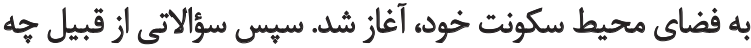

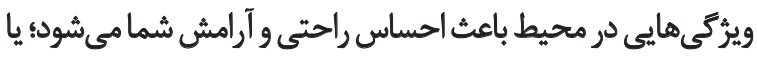

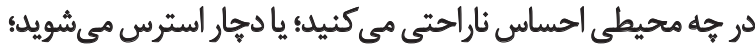

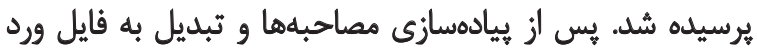

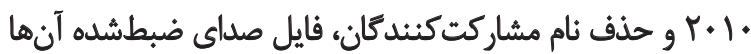

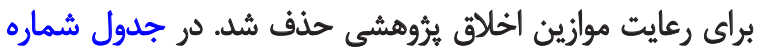

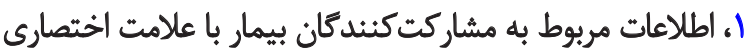

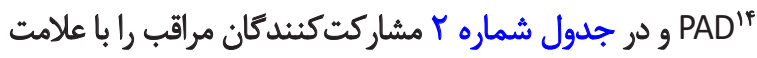

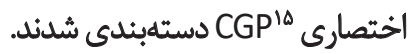

علاوهبر دشوارى جمعآورى اطلاعات صحيح از طريق مصاحبه

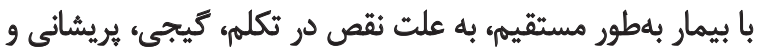

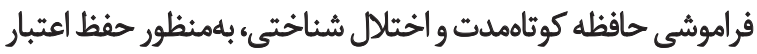

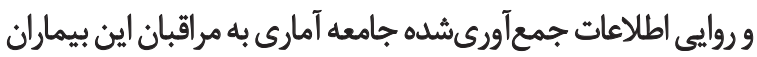

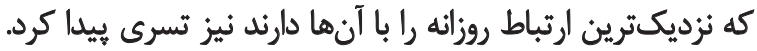

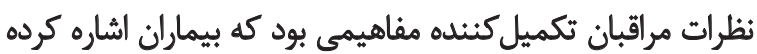

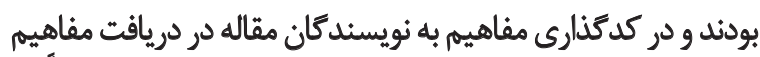

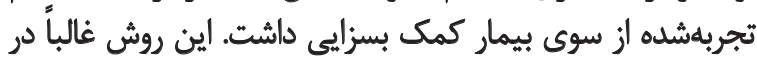

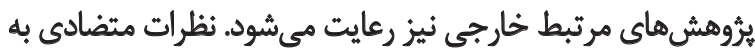

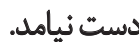

يثروهش حاضر يك مطالعه مرورى بر مبناى روش تحليل

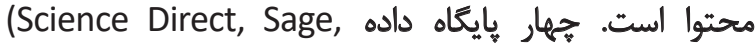
بيش إن (Springer, Content IOS press)

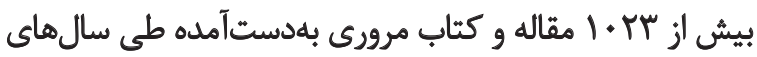

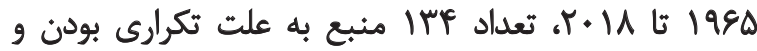

14. Participant with Alzheimer Disease (PAD)

15. Care Giver Participant (CGP)
يادمان، واقع در منطقه ب شهر تهران انتخاب شديند. اين كلينيك يكى

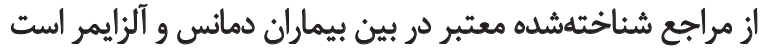

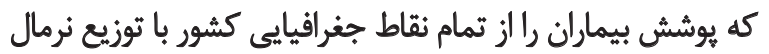

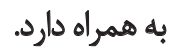

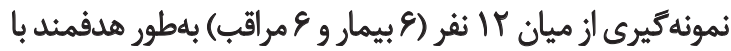

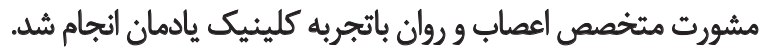

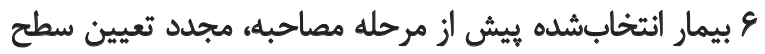

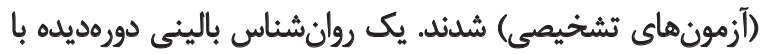

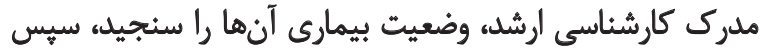

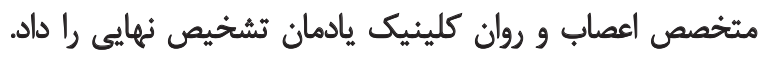

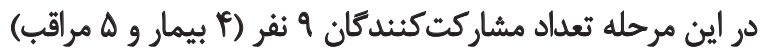

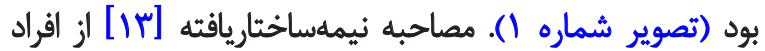
انتخابشده به مدت هار اتا ·r دقيقه انجام شد.

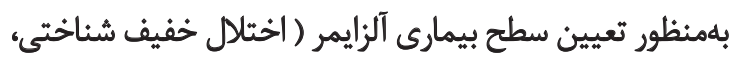

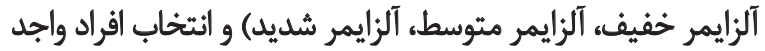

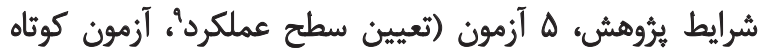

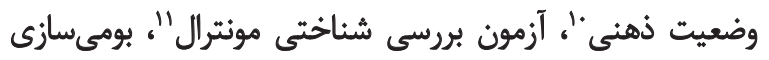

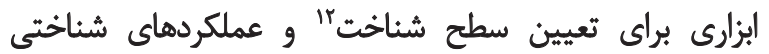

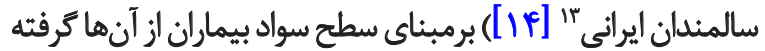

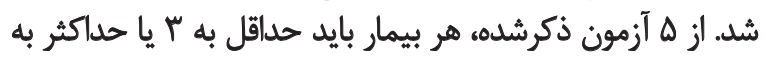

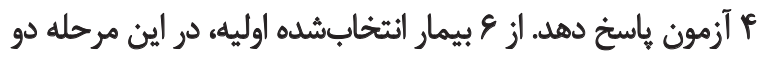

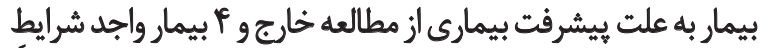
مصاحيه انتخغاب شئلئ.

9. Functional Assessment Staging (FAST)

10. The Mini Mental State Examination (MMSE)

11. Montreal Cognitive Assessment (MoCA)

12. Cognition

13. Persian Test of Elderly for Assessment of Cognition and Executive function (PEACE) 


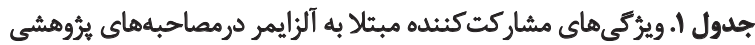

\begin{tabular}{|c|c|c|c|c|c|c|c|c|c|c|c|}
\hline \multicolumn{5}{|c|}{ نمرات ارزيابى أزمون هاى تعيين سطح بيمارى } & \multirow{2}{*}{ سيمارى سطح } & \multirow{2}{*}{ ميمارى مدت } & \multirow{2}{*}{ شيغل } & \multirow{2}{*}{ تحميالات } & \multirow{2}{*}{ جنسيت } & \multirow{2}{*}{ سن } & \multirow{2}{*}{ شركت كثندكان } \\
\hline FAST & MMSE & MoCA & CFT & Bread & & & & & & & \\
\hline f & - & - & 1. & A. & FAST $F$ & fl & أزاد & إيى نسواد & هرد & Ar & PAD1 \\
\hline$f$ & Ir & - & - & $f$ & FAST $F$ & 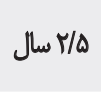 & ريير & فوق دييلم & مرد & 99 & PAD2 \\
\hline$p$ & 10 & - & - & $r$ & FAST $Y$ & 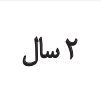 & 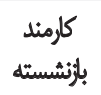 & سيكل قديهم & مرد & $n^{\mu}$ & PAD3 \\
\hline $\mathrm{MCl}$ & - & $\mathrm{M}$ & 1. & - & $\mathrm{MCl}$ & r بال & تخصوق تراتحي & تخصصى دكتى & مرد & $w$ & PAD4 \\
\hline
\end{tabular}

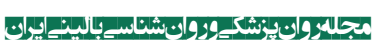

جدول T. ويرُكَى هاى مراقبان بيمار مشاركت كنثده در مصاحبهها

\begin{tabular}{|c|c|c|c|c|c|}
\hline مدت مرأقبت & نسبت با بيمار & تحصيلات & جنسيت & سن & مشاركت كتندكان (مراقيان) \\
\hline 1/ه سال & يريشتار شبائه روزى & 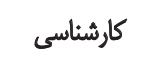 & مرد & $r$ & $\mathrm{CP} 1$ \\
\hline 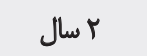 & همسر بيمار & كارشناسى ارشد & زن & rq & $\mathrm{CP} 2$ \\
\hline 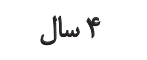 & ل يسر بيمار & فوق دييلم فنى & 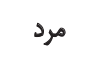 & m & CP3 \\
\hline 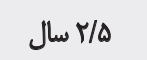 & همسر بيمار & 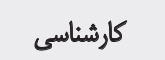 & زن & s. & $\mathrm{CP} 4$ \\
\hline r بال & همسر بيمار & كارشناسى ارششد & زن & gr & CP5 \\
\hline
\end{tabular}

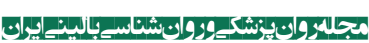

توسط يك نويسنده"، يكى ديكر از نويسئدكان بهطور جداكانه

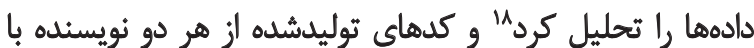

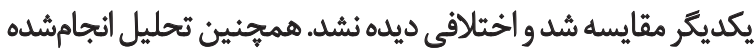

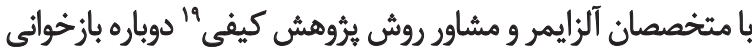

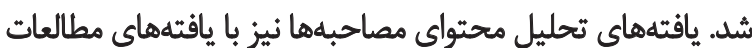
مشابه مقايسه شد.

batiols

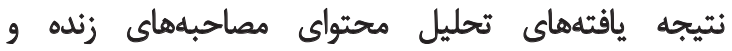

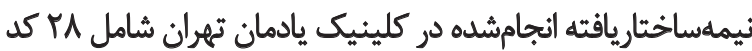

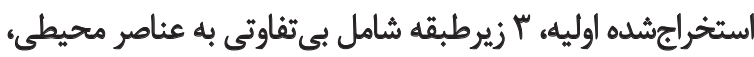

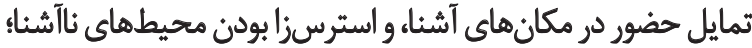

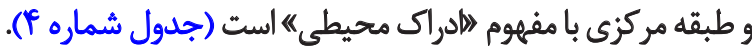

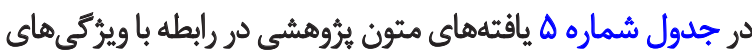

17. Member check

18. Peer check

19. Expert check
مشابهت زياد از مطالعه خارج شد. يّ از مرور خكيده

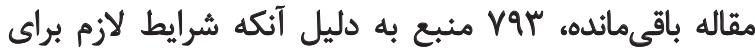

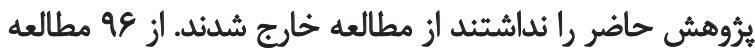

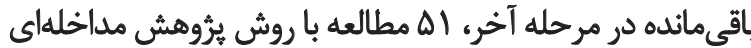

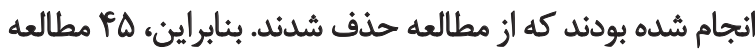

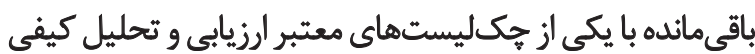

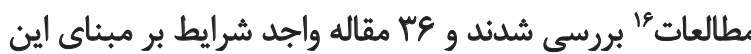

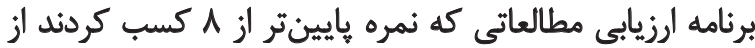

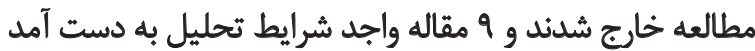

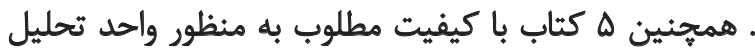

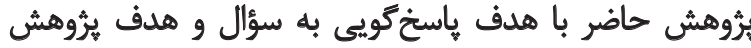

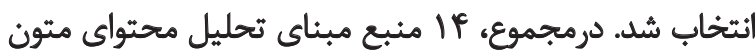
در بخش دوم انتخاب شد (جدول شماره س).

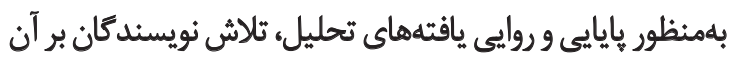

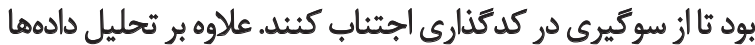

16. Critical Appraisal Skills Program Checklist (CASP) 
جدول r. ارزيابي مطالعات واردشده بر مبناى جك ليست CASP

\begin{tabular}{|c|c|c|c|c|c|c|c|c|c|c|}
\hline \multicolumn{11}{|c|}{ يرسشها } \\
\hline 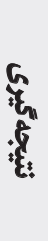 & 焉 & $\begin{array}{l}\frac{5}{8} \\
\frac{6}{6} \\
\frac{6}{8}\end{array}$ & 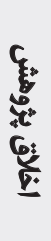 & 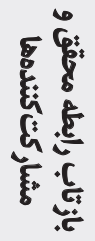 & 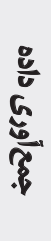 & $\frac{G}{G}$ & 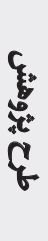 & $\frac{\xi}{\varepsilon}$ & 6. & نويسند كان و سال نُشر \\
\hline+ & + & + & + & + & + & + & - & - & + & 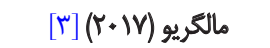 \\
\hline+ & + & + & - & + & + & - & + & + & + & استرنبرك (•|+r) [ع|] \\
\hline+ & + & + & + & - & + & + & + & + & + & ابرهارد (9+ + (T) [1Q] \\
\hline+ & + & + & + & + & + & + & + & + & + & 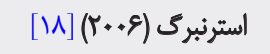 \\
\hline+ & + & + & + & + & + & - & + & + & + & 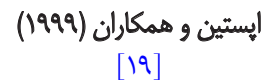 \\
\hline+ & + & + & + & + & + & + & + & + & + & زيسل (Y..r) [•r] \\
\hline+ & + & + & + & - & + & + & + & + & + & 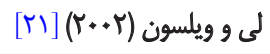 \\
\hline+ & + & - & - & + & + & + & + & + & + & 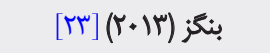 \\
\hline+ & + & + & + & + & + & + & + & + & - & ادلستين و همكاران (ه+ + (T) \\
\hline+ & + & + & + & + & + & + & - & - & + & تيوب (194) [ب] \\
\hline+ & + & + & + & - & + & + & - & + & + & كنورسكى (19QV] \\
\hline+ & + & + & - & - & + & + & + & + & + & مريمن و همكاران (Y.1\%) \\
\hline+ & + & + & + & + & + & + & + & + & + & كولير و توومن (1) + [TV] \\
\hline+ & + & + & + & - & + & + & + & - & + & 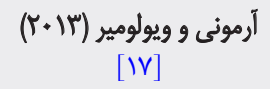 \\
\hline
\end{tabular}

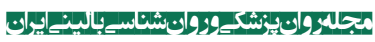

و غناي مفهومى بيشترى دارد، يافتههاي تحليل محتواي حاصل

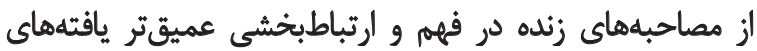

يروهشهاى كذشته نقش بسزايى دارد.

اين يافتهها در مقايسه و انطباق ميان ويزّكى هاي محيط كالبدى

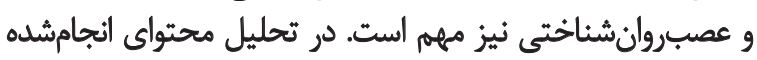

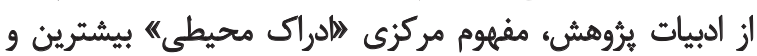

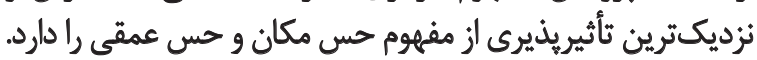

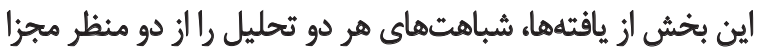

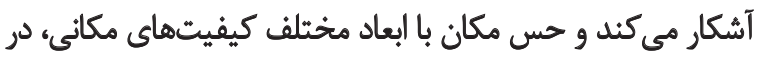

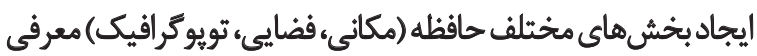

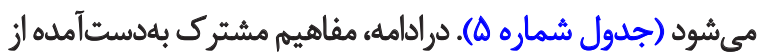

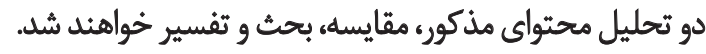

محيط كالبدى و عصبروانشئاختي مبتلايان به دمانس از نوع

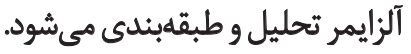

ثै يافتههاى حاصل از تحليل محتواى مصاحبههاى

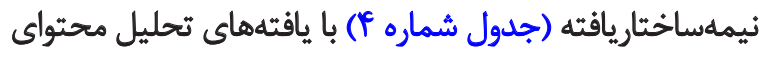

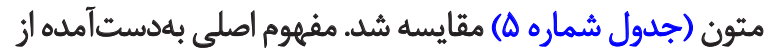

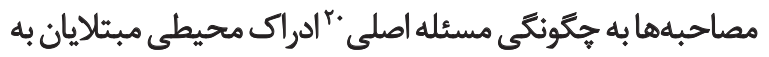

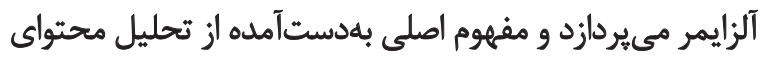

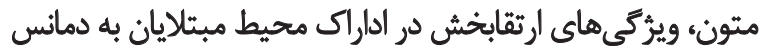

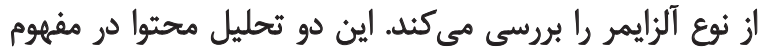

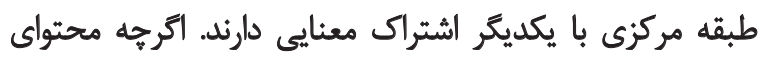

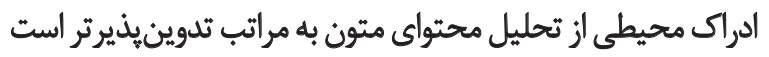


جدول f. تحليل محتواى حاصل از مصاحبههاي زئده از مبتلايان به آلزايمر و مراقبان آنها

\begin{tabular}{|c|c|c|}
\hline مفوم (طبقه) سطح) & مفهوم سطح دوم (زيرطبقه) & مفهوم سطح اول (كد) \\
\hline ادراك هحيطي & جنى تنفاوتى نسبت بحيطي & 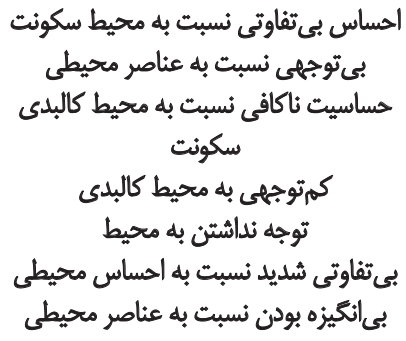 \\
\hline
\end{tabular}

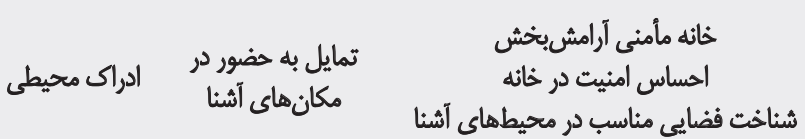

استرسزا بودن ادراك محيطى محيطهاي نأiٔشنا
استرس در محيط هاي جديل، ناشناخته و

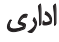
احساس نالشنائى محيط ناشى از تنها مائلن

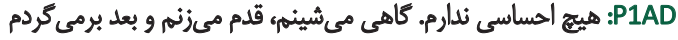

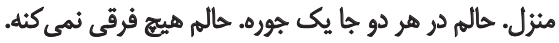

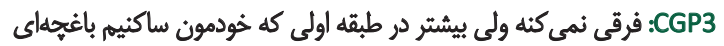

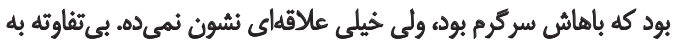
هحيط. CGP2

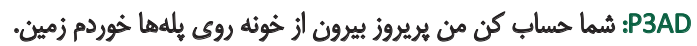

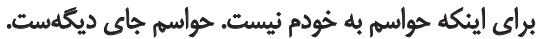

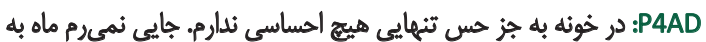

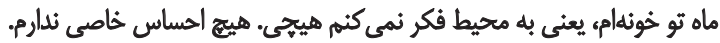

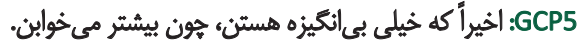

CGP3

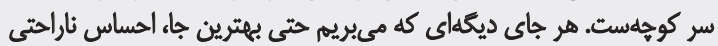

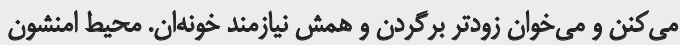

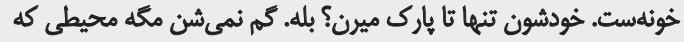

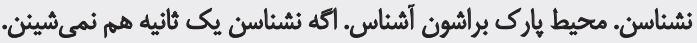

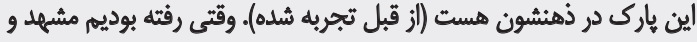

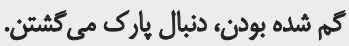

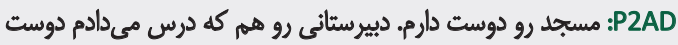

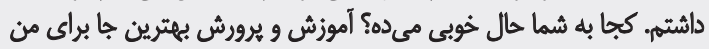

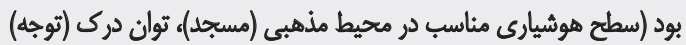

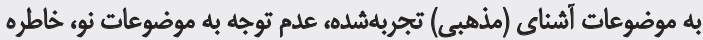

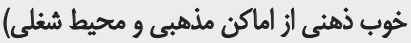
CGP1

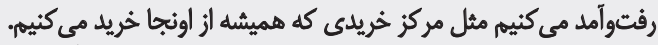
CGP2

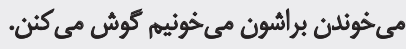

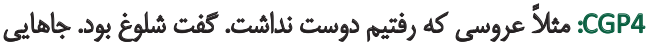

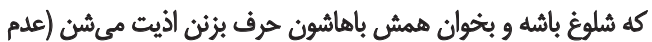

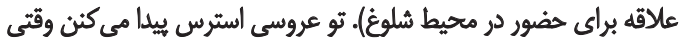

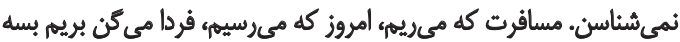

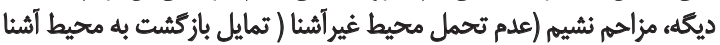

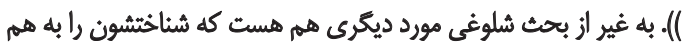

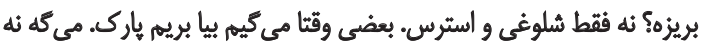

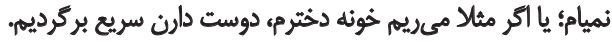

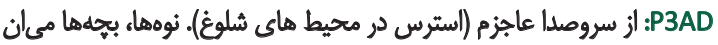

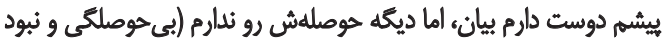

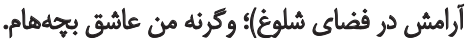

CLP1

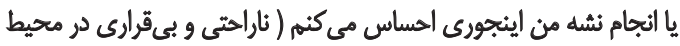

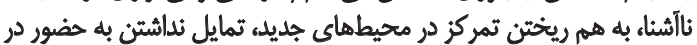
محيطهاي نالآشنا).

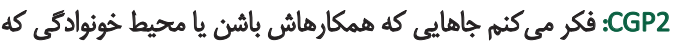

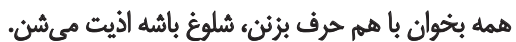
CGP3

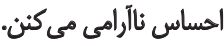

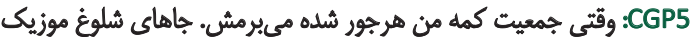

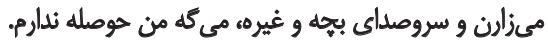


جدول هـ. بررسى ويركى هاى محيط كالبدى و عصبشناختى براى مبتلايان به دمانس از نوع آلزايمر

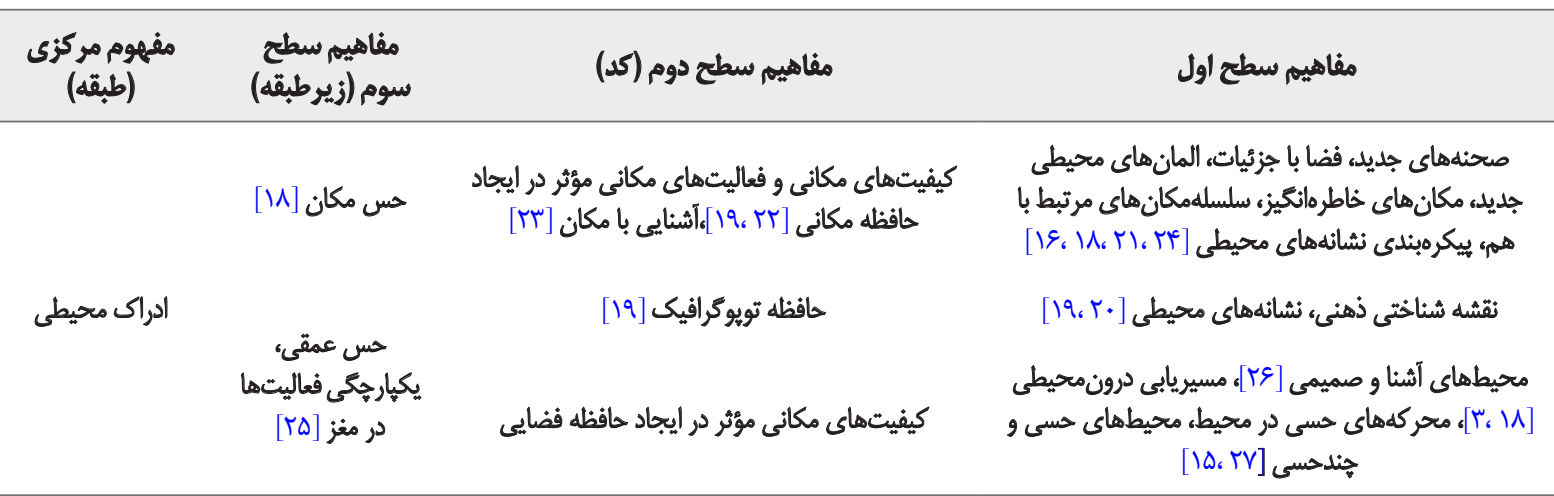

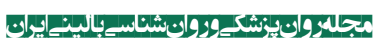

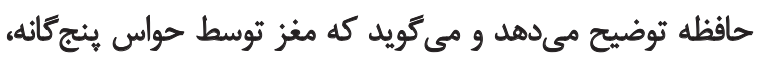

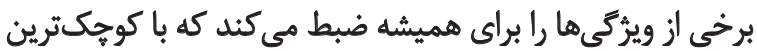

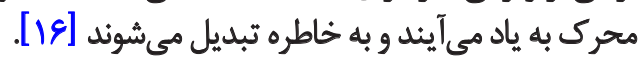

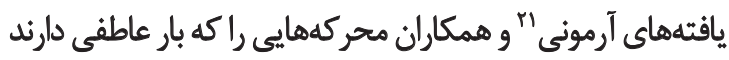

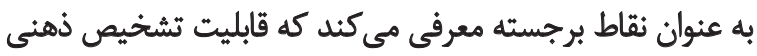

21. Armony

\section{"ابى تثاوتى به عناصر محيطى" و امحركه هاى حسى"} ابرنارد بسيارى از عوامل محيطى را تأثيركذار بر مغز و جسم انسان

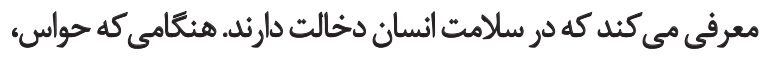

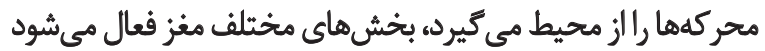

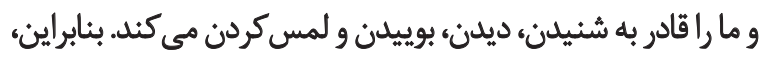

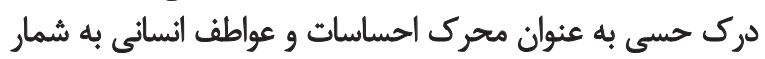

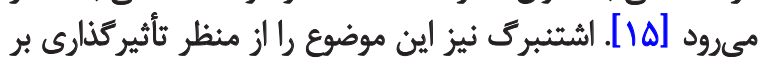

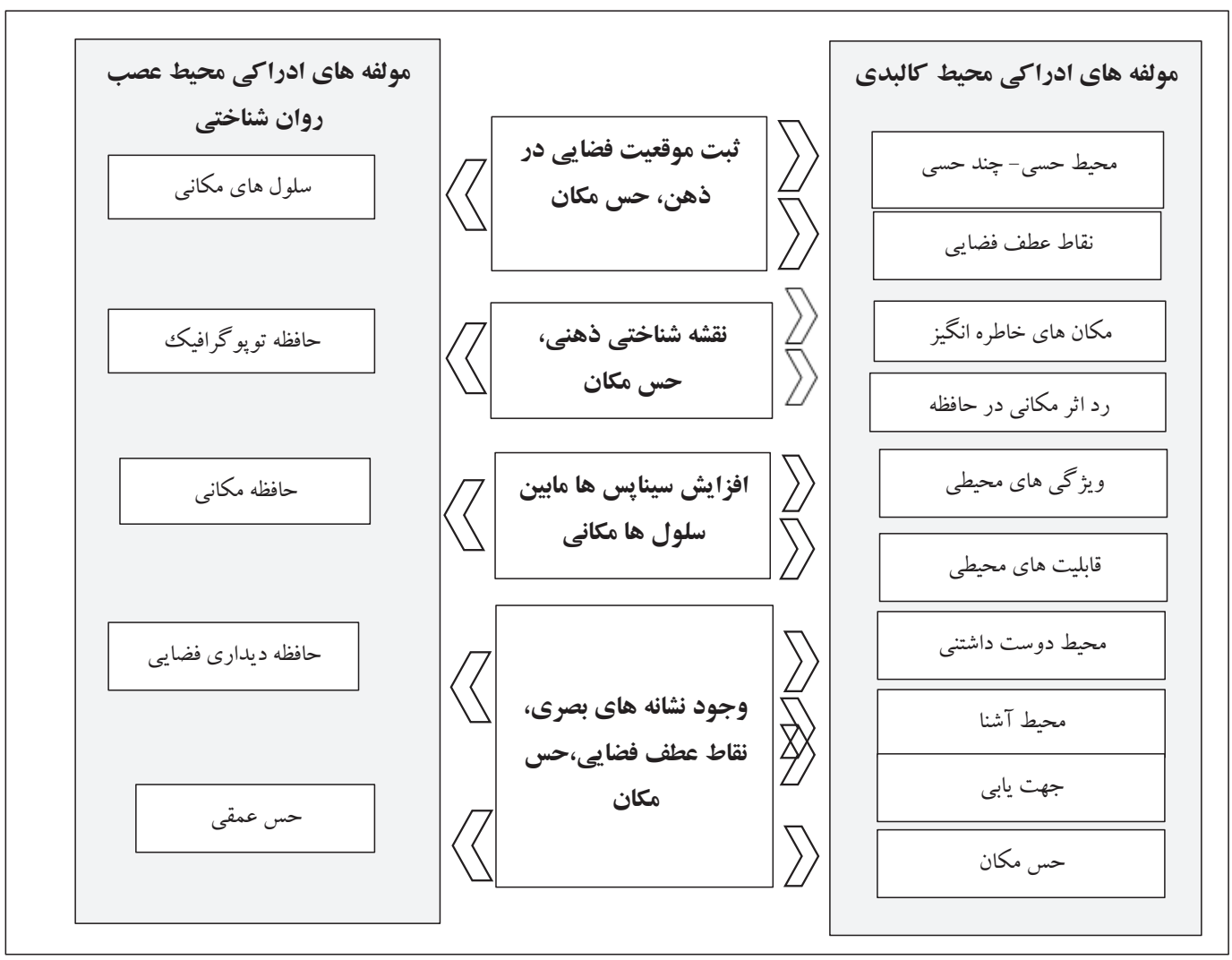

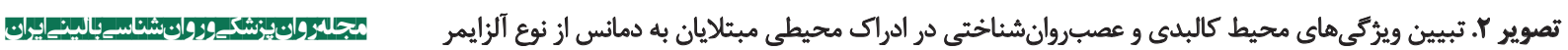


آنجهه كه در آن مكان ياد كرفته است رابطه برقرار كند؛ يعنى، خاطره

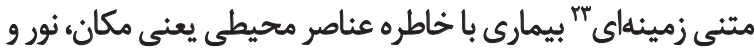

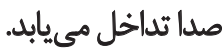

فرد مبتلا به علت كم كشتكى و نشناختن ساختمانها، اشياى بـاي

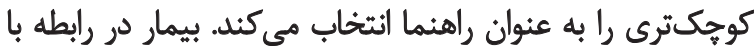

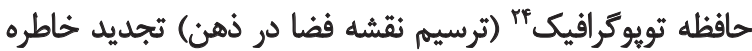

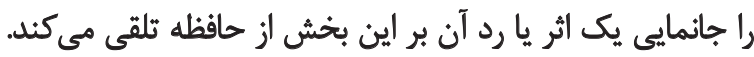

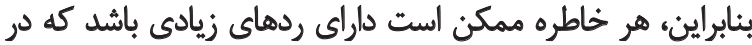

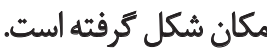

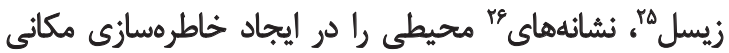

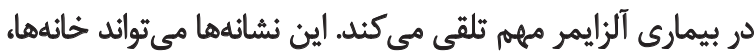

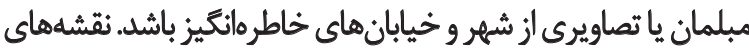

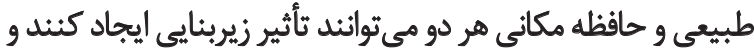

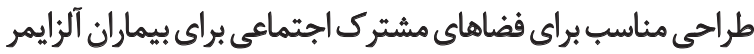

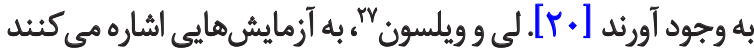

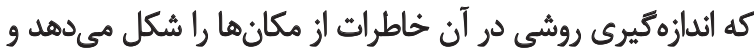

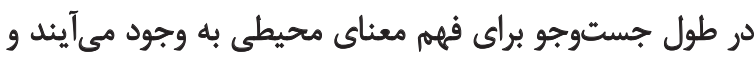

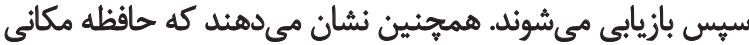

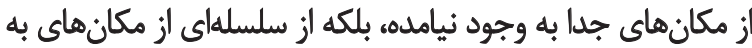

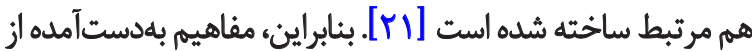

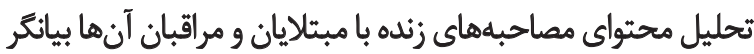

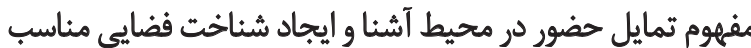

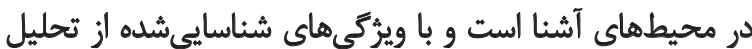
محتواى متونى قابل تطبيق است كه معرف كيفيتهاي مانى مكانى مؤثر

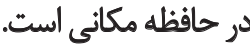

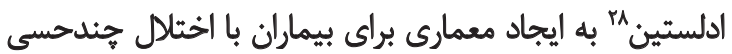

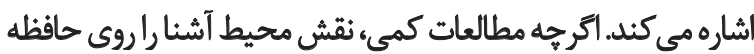

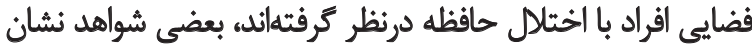

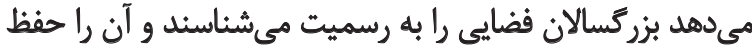

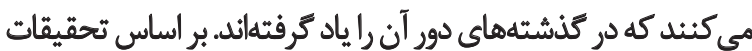

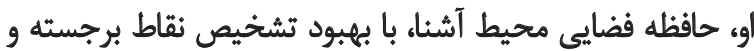

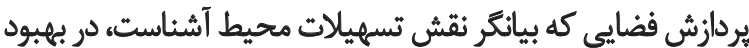

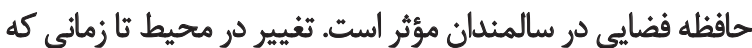

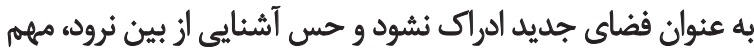

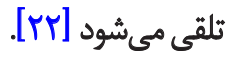

آشنايى فرد مبتلا با محيط، يكى ديكر از شباهتهاى بهدستآمده

23. Conceptual memory

24. Topographic memory

25. Ziesel

26. Cues

27. Lee \& Wilson

28. Edelstein

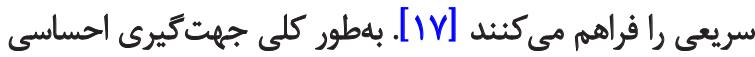

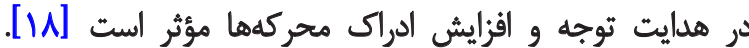

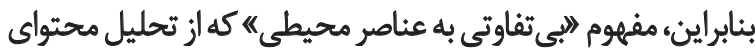

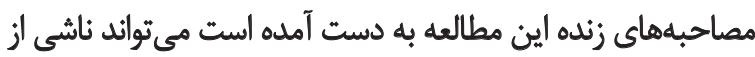

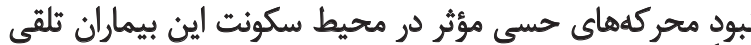
شود.

در معمارى مبتنى بر عصبروانشناختى لامعمارى حسى بهمثابه

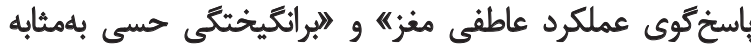

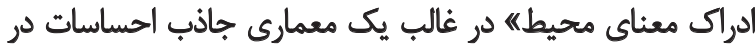

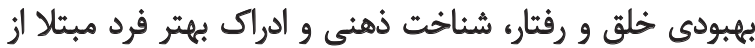

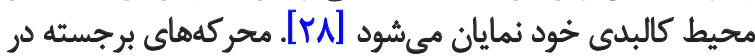

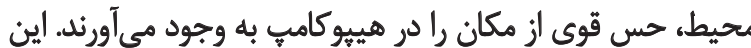

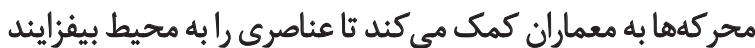

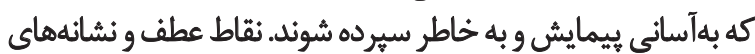

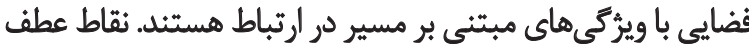

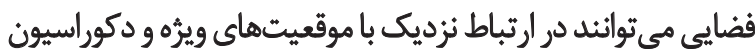

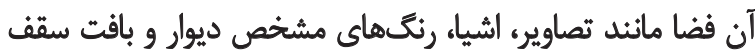

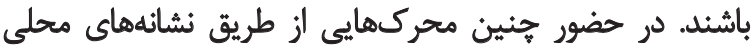

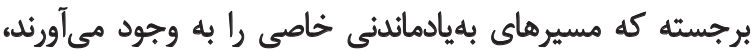

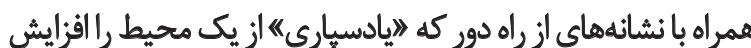

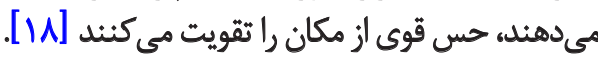

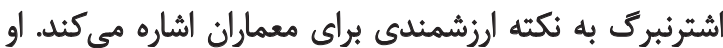

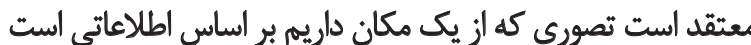

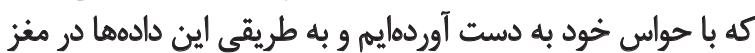

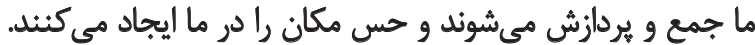

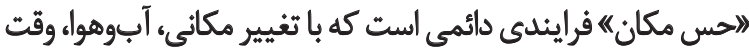

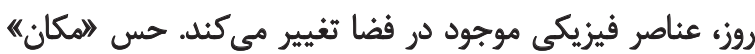

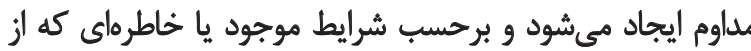

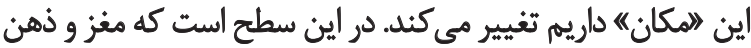

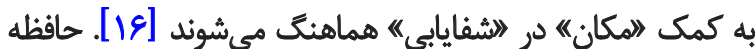

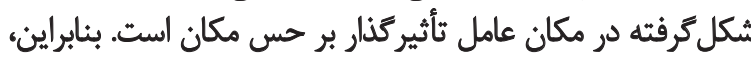

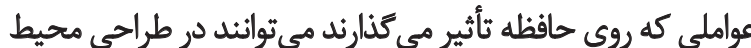

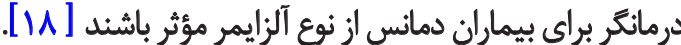

"تمايل حضور در مكانهاي آشناه و "حافظه مكانى و فضايى" بر اساس مطالعات اشتنبرى "ه در بيمارى آلزايمر حافظه مكان از

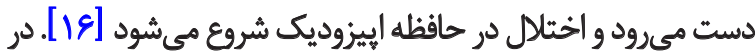

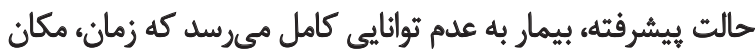

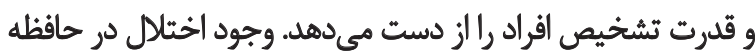

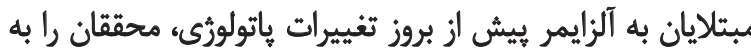

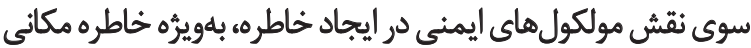

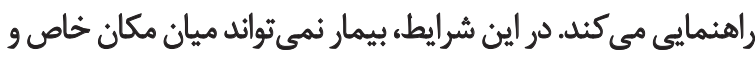

22. Stenberg 
ثائوب" به نشانههاى بصرى مسير اشاره مي كند كه ممكن است

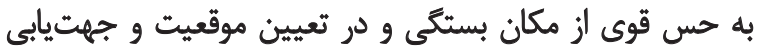

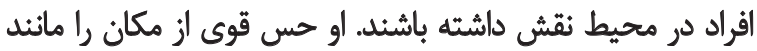

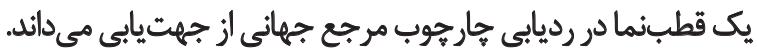

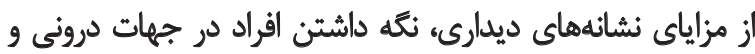

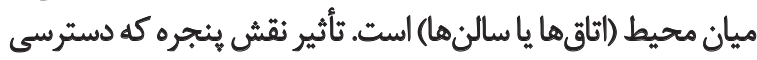

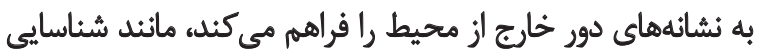

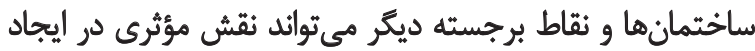

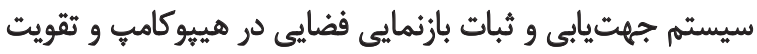

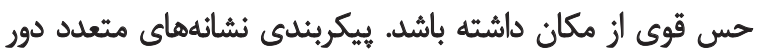

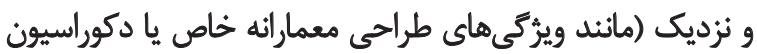

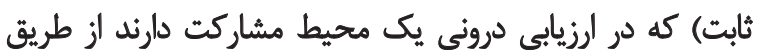

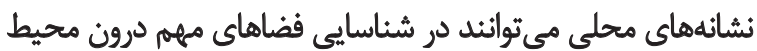

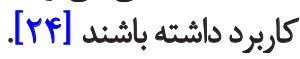

اشترنبرك، مغز را براى هدايت جهتيابى نيازمند حس عمقى

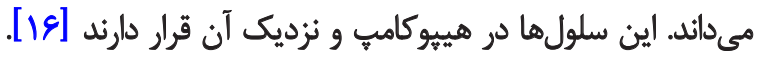

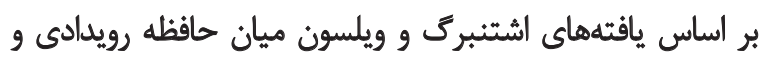

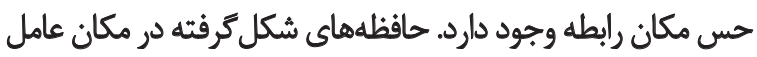

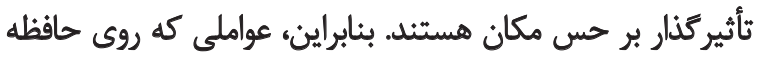

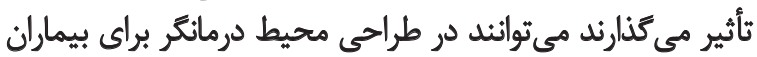

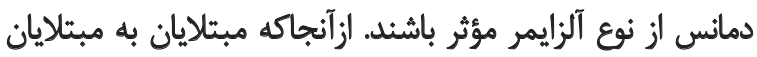

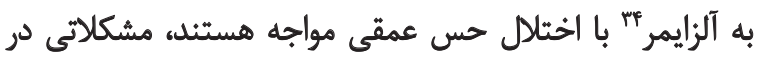

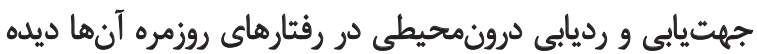

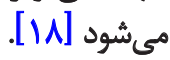

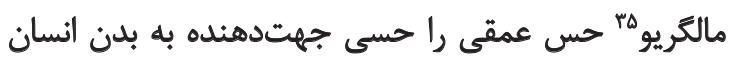

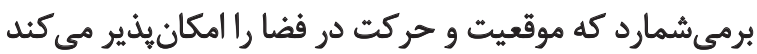

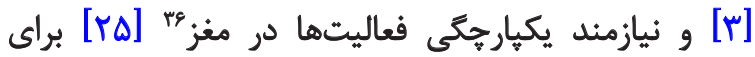

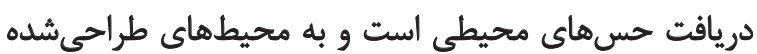

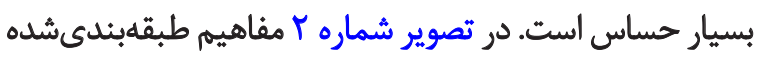

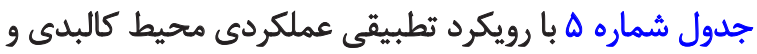

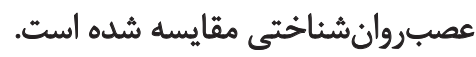

\section{تيجيجئيرى}

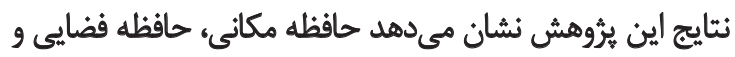

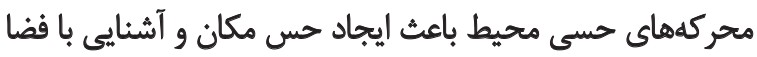

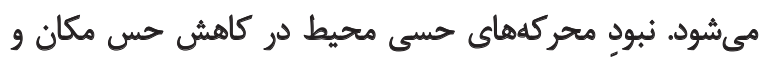

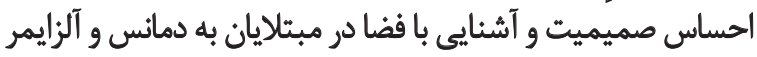

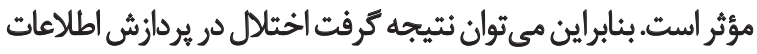
در حافظه مكانى و فضايى (عوامل درونى) در اين بيماران و نبود بردان

32. Taube

33. Internal clues

34. Dementia Alzheimer Type (DAT)

35. Mallgrave

36. Integrative activity of the brain
از هر دو تحليل محتواي انجامشده در اين بروهش است. اشتنبرى و و

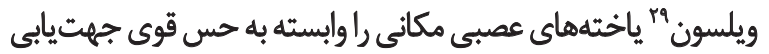

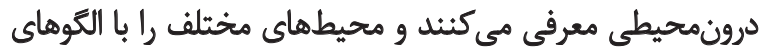

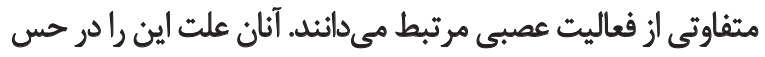

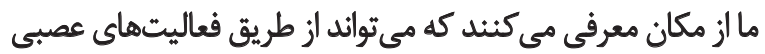

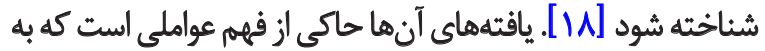

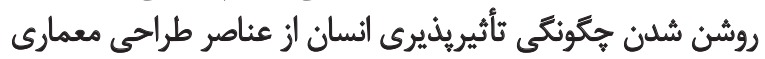
وابسته است.

\section{(استرسزا بودن محيطهاى ناآشناش و "حافظه مكانى و \\ فضايى"}

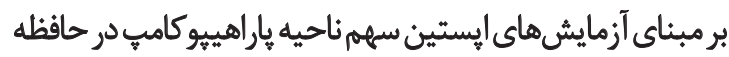

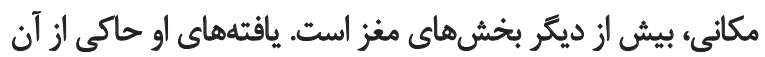

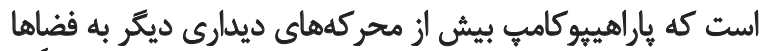

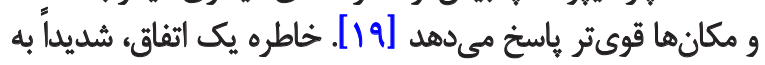

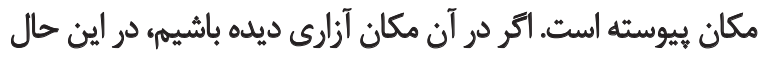

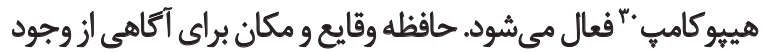

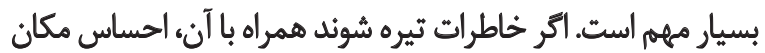

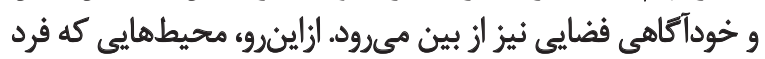

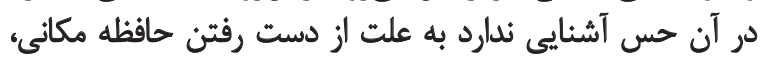

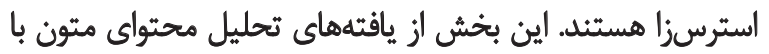
تحليل مصاحبههاى زنده منطبق است.

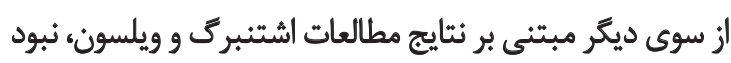

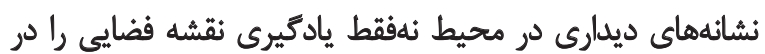

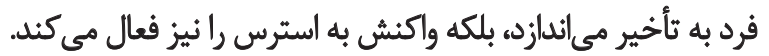

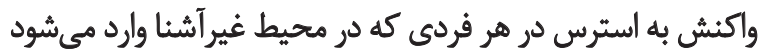

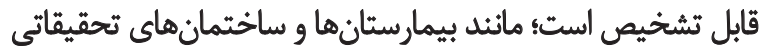

كه راهروهاي طولاتى، بدون نشانهاي ديداري دارند [1/1]].

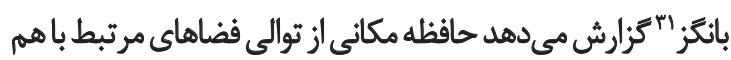

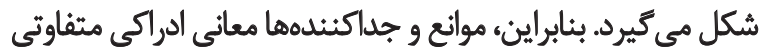

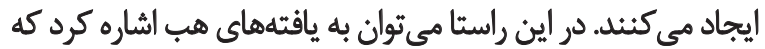

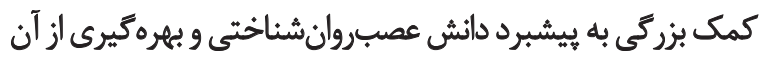

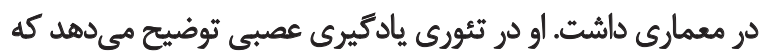

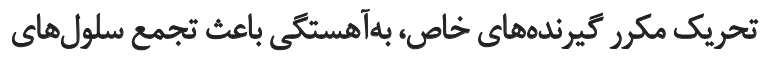

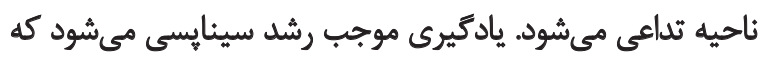

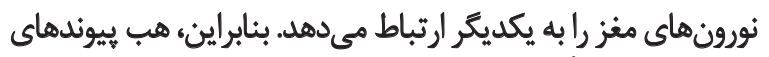
عصبى را عامل تأثير كذار در ادراك مى داند.

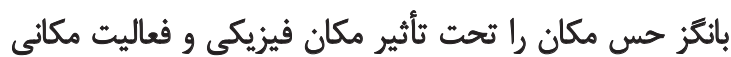

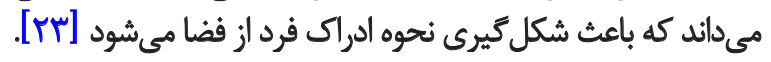

29. Stenberg \& Wilson

30. Hippocampus

31. Bangs 


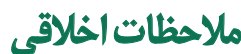 \\ ييروى از اصول اخالاق يثروهشي}

در اين يثروهش براى رعايت موازين اخلاقي يثوهش (محر مانه بودن

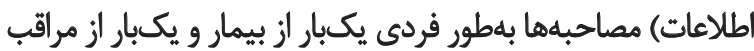

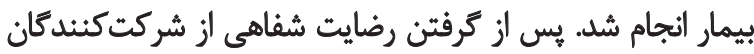

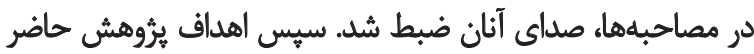

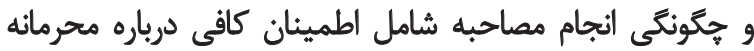

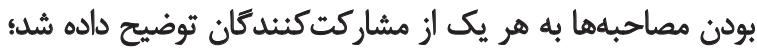

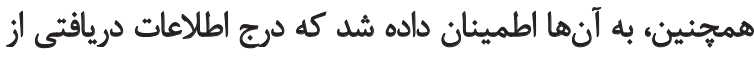

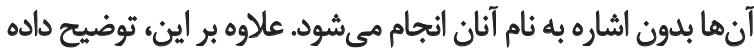

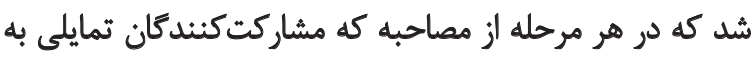

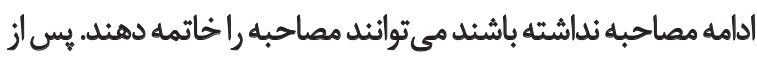

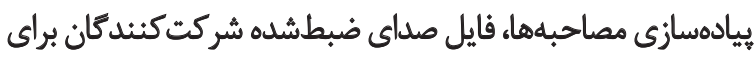

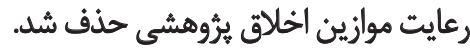

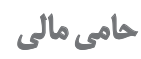

اين مقاله از إياننامه مقطع دكتراى نويسئده اول در دانشعاه علهم

$$
\text { و صنعت ايران گرفته شده است. }
$$

$$
\text { مشاركت نويسندكان }
$$

مشاركت نويسندكان مقاله به اين شرح است: مفهومسازي: سيما

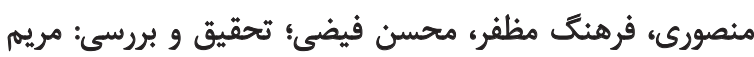

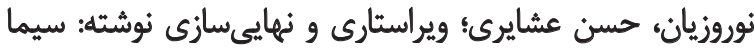

منصورى، مريم نوروزيان. - منئ.

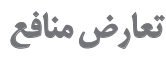

بنا به اظهار نويسئده مسئول، اين مقاله تعارض منافع ندارد.

$$
\text { سياسكَّز ارى }
$$

بدينوسيله از كاركنان كلينيك يادمان به دليل همكارى با اين

$$
\text { ئروهش صميمانه قدردانى مى كارود. }
$$

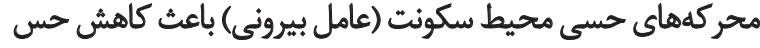

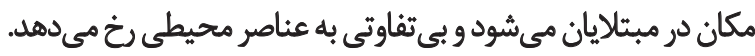

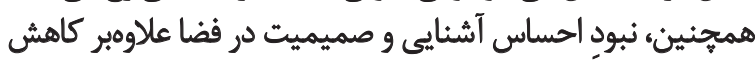

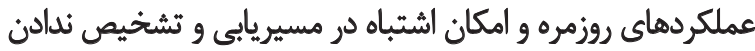

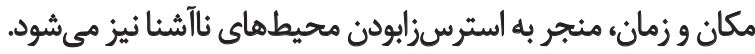

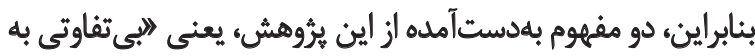

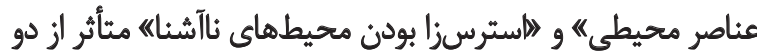

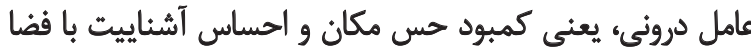

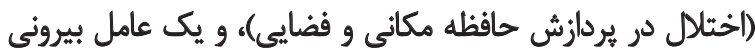

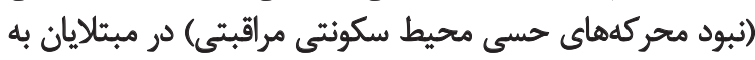

$$
\text { دمانس است. }
$$

از سوى ديكر، مؤلفههاى محيط كالبدى و عصبروانشئاختى

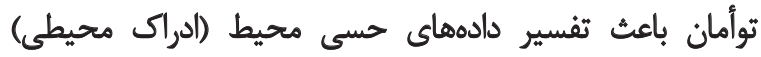

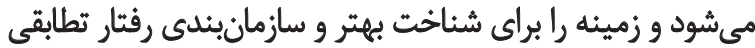

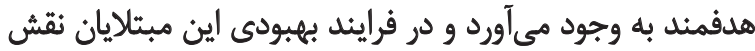

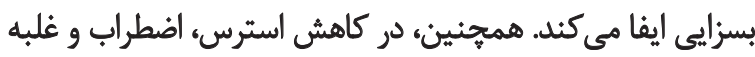

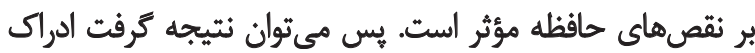

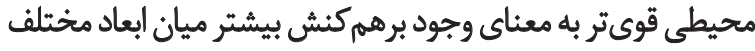

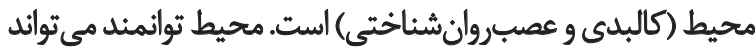

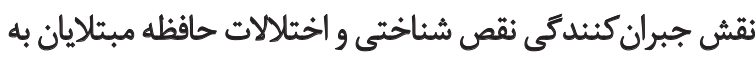

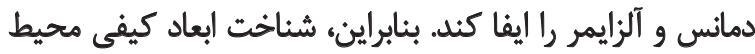

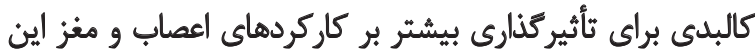

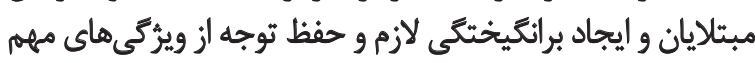

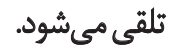

سلولهاى عصب حسى مغز تحت تأثير ويرُّى هاى شكل دهنده

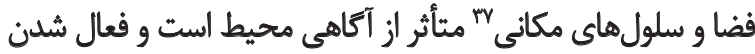

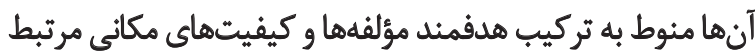

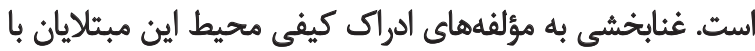

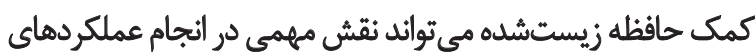

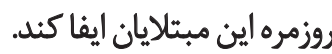

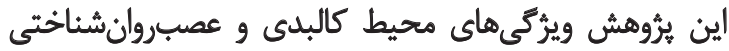

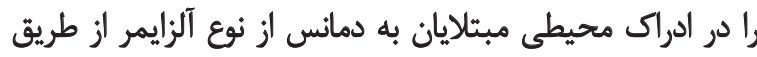

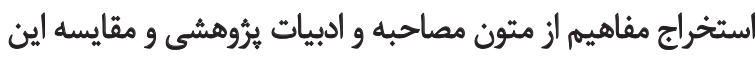

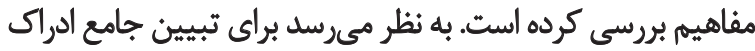

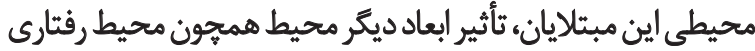

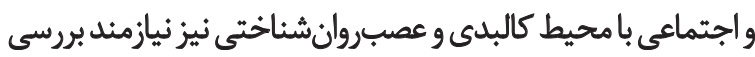

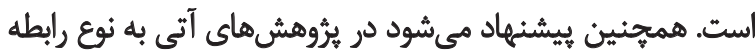

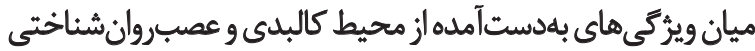
در ادراك محيطى مبتلايان به دمانس از نوع آلزايمر برداخته شودان

37. Place neuron 


\section{References}

[1] Sung HC, Chang AM. Use of preferred music to decrease agitated behaviors in older people with dementia: A review of the literature. Journal of Clinical Nursing. 2005; 14(9):1133-40. [DOI:10. 1111/j. 1365-2702. 2005. 01218. x] [PMID]

[2] Lawton MP. The physical environment of the person with Alzheimer's disease. Aging \& Mental Health. 2001; 5(supl. 1):56-64. [PMID]

[3] Mallgrave HF. The Architects Brain: Neuroscience, Creativity, and Architecture [ K Mardomi, S Ebrahimi, Persian trans.]. Tehran: Honar-E Memari Publication; 2017.

[4] Brocki KC, Bohlin G. Executive functions in children aged 6 to 13: A dimensional and developmental study. Developmental Neuropsychology. 2004; 26(2):571-93. [DOI:10. 1207/ s15326942dn2602_3] [PMID]

[5] Kaplan S, Berman MG. Directed attention as a common resource for executive functioning and self-regulation. Perspectives on Psychological Science. 2010; 5(1):43-57. [DOI:10. 1177/1745691609356784] [PMID]

[6] Kaplan S. The restorative benefits of nature: Toward an integrative framework. Journal of Environmental Psychology. 1995; 15(3):169-82. [DOI:10. 1016/0272-4944(95)90001-2]

[7] Atchley P, Warden AC. The need of young adults to text now: Using delay discounting to assess informational choice. Journal of Applied Research in Memory and Cognition. 2012; 1(4):229-34. [DOI:10.1016/j. jarmac. 2012. 09. 001]

[8] Biren, AB. Spatial manifestations of the human psyche. Paper presented at: 2014 ANFA Conference. 23 July 2014; San Diego, California.

[9] Pasqualini I, Blanke O. The architectonic self: own body perception and feelings in architectonic space. Cognitive Processing. 2012; 13: S27-S27.

[10] Sarmad Z, Bazargan A, Hejazi E. [Research methods in behavioral sciences (Persian)]. Tehran: Agah; 2015.

[11] Heydari SH. [Introduction to research method in architecture (Persian)]. Tehran: Fekre Now; 2014.

[12] Cho JY, Lee EH. Reducing confusion about grounded theory and qualitative content analysis: Similarities and differences. The Qualitative Report. 2014; 19(32):1-20.

[13] Delavar, A. [Theoretical and practical principle of research in humanities and social sciences (Persian)]. Tehran: Roshd; 2013.

[14] Javadi PS, Zendehbad A, Darabi F, Khosravifar S, Noroozian M. Development and implementation of Persian test of Elderly for Assessment of Cognition and Executive function (PEACE). Electronic Physician. 2015; 7(7):1549-56. [DOI:10. 19082/1549]

[15] Eberhard JP. Applying neuroscience to architecture. Neuron. 2009; 62(6):753-6. [DOI:10. 1016/j. neuron. 2009. 06. 001] [PMID]

[16] Sternberg EM. Healing Spaces: The Science of Place and WellBeing. Cambridge: Harvard University Press; 2010.

[17] Armony J, Vuilleumier P. The Cambridge handbook of human affective neuroscience. Cambridge: Cambridge University Press; 2013. [DOI:10. 1017/CBO9780511843716]
[18] Sternberg EM, Wilson MA. Neuroscience and architecture: Seeking common ground. Cell. 2006; 127(2):239-42. [DOI:10. 1016/j. cell. 2006. 10. 012] [PMID]

[19] Epstein R, Harris A, Stanley D, Kanwisher N. The para hippocampal place area: Recognition, navigation, or encoding? Neuron. 1999; 23(1):115-25. [DOI:10. 1016/S0896-6273(00)80758-8]

[20] Zeisel J. Evidence-based design in coordinated health treatment. Journal of Evidence-Based Design as Health Treatment $2003 ; 35-43$

[21] Lee AK, Wilson MA. Memory of sequential experience in the hippocampus during slow wave sleep. Neuron. 2002; 36(6):118394. [DOI:10. 1016/S0896-6273(02)01096-6]

[22] Edelstein RS, Ghetti S, Quas JA, Goodman GS, Alexander KW, Redlich $\mathrm{AD}$, et al. Individual differences in emotional memory: Adult attachment and long-term memory for child sexual abuse. Personality and Social Psychology Bulletin. 2005; 31(11):1537-48. [DOI:10. 1177/0146167205277095] [PMID]

[23] Bangs L. Psychotic reactions and carburetor dung: The work of a legendary critic: Rock' $\mathrm{N}^{\prime} \mathrm{Roll}$ as literature and literature as Rock'N'Roll. New York: Anchor; 2013.

[24] Taube JS. Head direction cells and the neurophysiological basis for a sense of direction. Progress in Neurobiology. 1998; 55(3):22556. [DOI:10. 1016/S0301-0082(98)00004-5]

[25] Konorski J. Integrative activity of the brain. Chicago: The University of Chicago Press; 1967.

[26] Merriman NA, Ondřej J, Roudaia E, O'Sullivan C, Newell FN. Familiar environments enhance object and spatial memory in both younger and older adults. Experimental Brain Research. 2016; 234(6):1555-74. [DOI:10. 1007/s00221-016-4557-0] [PMID]

[27] Collier L, Truman J. Exploring the multi-sensory environment as a leisure resource for people with complex neurological disabilities. NeuroRehabilitation. 2008; 23(4):361-7. [PMID]

[28] Mansoori S, Faizi M, Ashayeri H. [New discourse in architecture; Architecture of Neuropsychology (Persian)]. Soffeh. 2018; 28(80):25-40. 